\title{
Product Discrimination system using deep learning
}

\author{
Hitoshi Nakao, Taku Akase, Lifeng Zhang \\ Kyusyu Institute of Technology, Kitakyusyu, 804-8550, Japan \\ *Corresponding Author: zhang@ele.kyutech.ac.jp
}

\begin{abstract}
Nowadays, systems that use AI technology (artificial intelligence technology) and image processing technology have been introduced to factories, and are used in the logistics industry to eliminate labor shortages and prevent human errors. However, the existing image processing technology cannot identify the product name from the package image because the characters printed on the package are distorted and their colors and fonts are not uniform. Therefore, we propose a system previously which enabled this task using character recognition technology by Deep Learning with a certain accuracy. In this research, we focus on how to make a image processing which can obtains an high accuracy, and show how much the recognition accuracy is improved. Our results reveal that the recognition rate is improved by the proposed method.
\end{abstract}

Keywords: Deep Learning, classification system, image processing.

\section{Introduction}

In the logistics field, there is a process to confirm whether the product to be shipped is correct. In the past, this work was done by visual inspection, but it caused mis-shipment due to human error such as misinterpretation or assumption. Therefore, by introducing a system using computer image processing, this work will be made efficient or automated. In this study, the product is identified by reading the product name printed on the package from the product image. In traditional character recognition, we mainly use optical character recognition (OCR), but we use deep learning to cope with character distortion. In recent years, deep learning of various algorithms has been developed and adopted for character recognition. Character recognition accuracy has dramatically improved, but noise in the characters and background prevents improvement in recognition accuracy. Characters of the product are not uniform in color or font, and there is noise due to the background color and light reflection. So the existing character recognition technology causes false recognition. In this paper, we examined how the recognition accuracy changes by performing image processing on the package image when recognizing. The proposed image processing technology is constructed using discriminant analysis which is one of binarization, and we use a handwritten character recognition data set called Mnist for character recognition.

\section{Principle}

\subsection{Discriminant analysis method}

Discriminant analysis method is a method of automatically performing binarization by finding the threshold at which the value of separation is maximum in a histogram. The degree of separation is the value at which the variance ratio between the between-class variance and the within-class variance is maximized. The within-class variance is the mean of the variances of each class, and the between-class variance is the variance of the mean of each class. Figure 1 shows the histogram of an image. A certain threshold $\mathrm{T}$ is determined from the histogram.

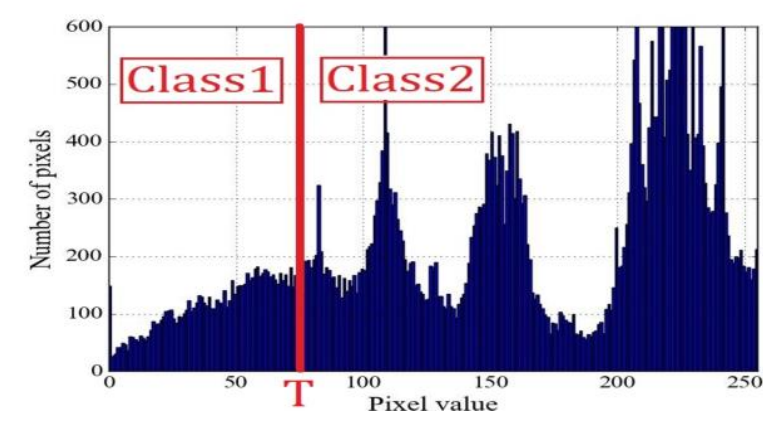

Fig 1. Histogram

\subsection{Denoising method using continuity of pixel values}

The proposed method removes background noise from the 
image subjected to discriminant analysis. Characters of a product image using discriminant analysis have continuous pixel values, so their characteristics are used to remove noise. If the pixel values are continuous, the pixel values output the same value. And if the pixel values are not continuous, the pixel value is output to be 255 (white).

\section{Experiment}

The characters to be recognized in this study are printed on a transparent bag and the contents are the background. It is assumed that combinations of printed characters and contents of various colors are decided in advance.

Prepare and label product images, perform deep learning, and create a learning model. The product assumes it 14kinds in experiments. Figure 2 shows the image of the product which we used.
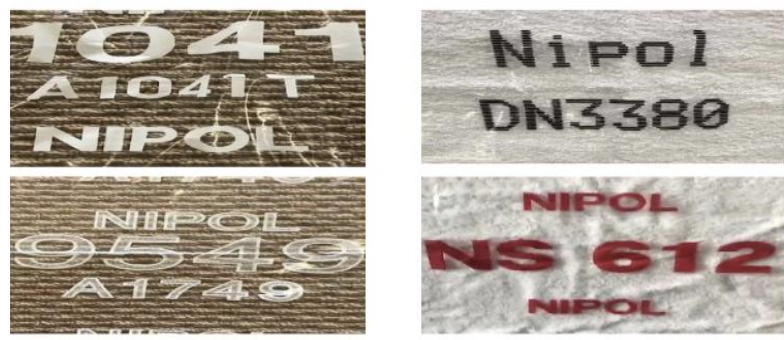

Fig. 2. Product examples

Figure 3 shows the image after the discriminant analysis method is performed on Figure 2.
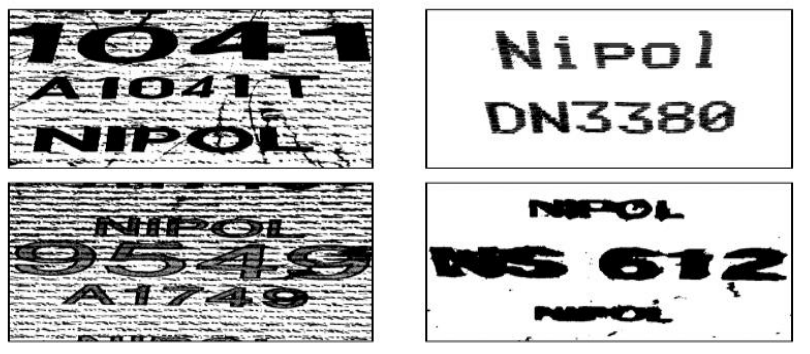

Fig. 3. Products performed to discriminant analysis

Figure 4 shows the image after performing the noise removal method introduced in principle on Figure 3.
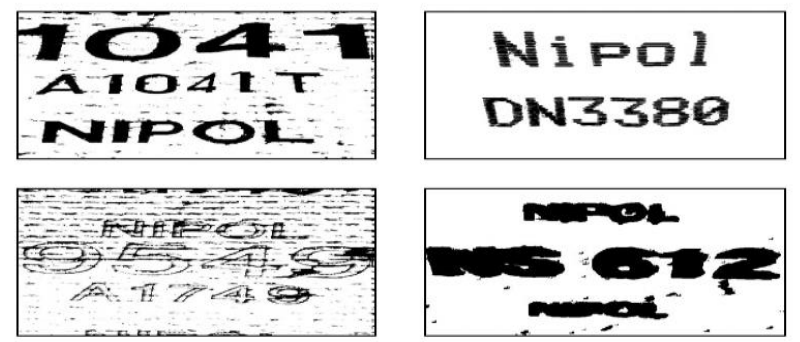

Fig. 4. Product performed the noise removal method The recognition rates of the characters in Figure 2, 3 and 4 became like Table 1.

Table 1. Recognition rate

\begin{tabular}{|l|c|c|c|}
\hline Figure & Fig. 2 & Fig. 3 & Fig. 4 \\
\hline Accuracy & $12 \%$ & $18 \%$ & $42 \%$ \\
\hline
\end{tabular}

The recognition rate has improved from $10 \%$ to $40 \%$. I think the reason for this is because the discriminant analysis method and the denoising method using the continuity of pixels make characters in the input image clear, and the character recognition system using deep learning can recognize the characters more correctly.

\section{Conclusions and Future Work}

In this paper, we have presented the method of binarization and denoising for character recognition system using deep learning. Our results show that recognition accuracy improves by using the proposed method. However, our results were only able to correctly recognize at recognition rate of $40 \%$, and there is still room for improvement. In addition, improvement for the practical use is necessary because a photography condition is severe. In the future, we would like to examine how much the recognition rate will improve by changing the image to be learned in deep learning, and the most suitable learning method to raise versatility.

\section{Acknowledgment}

The authors would like to acknowledge the support of Prof. Seiichi Serikawa, associate Prof. Akira Yamawaki, and assistant Prof. Yang Shiyuan.

\section{References}

(1) Yusuke Sugomori : "Deep Learning Java programing A theory and implementation of the depths Learning", Impress Corporation, pp. 121-131, 2016

(2) "Caffe", $<$ https://caffe.berkeleyvision.org/>

(3) "discrimination analysis method", < http://imagingsolution.blog.fc2.com/blog-entry-113.ht $\underline{\mathrm{ml}}>$

(4) "[image prcessing] discrimination analysis method Principle ,Feature ,Formula", $<$ https://algorithm.joho.in fo/image-processing/otsu-thresholding/ $>$ 